\title{
NLR - A Simple Indicator of Inflammation for the Diagnosis of Left Ventricular Hypertrophy in Patients with Hypertension
}

\author{
Xuefang Yu, ${ }^{1, *}$ MD, Yuchen Xue,,${ }^{1, *}$ MD, Bo Bian, ${ }^{1} \mathrm{MD}$, Xianming Wu, ${ }^{1} \mathrm{MD}$, Zhuoqun Wang, ${ }^{1} \mathrm{MD}$, \\ Jingjing Huang, ${ }^{1} \mathrm{MD}$, Lei Huang, ${ }^{1}$ MD and Yuemin Sun, ${ }^{1} \mathrm{MD}$
}

\begin{abstract}
Summary
We aimed to investigate the relationship between neutrophil-to-lymphocyte ratio (NLR), C-reactive protein (CRP), brain natriuretic peptide (BNP), and left ventricular hypertrophy (LVH) in hypertension. Methods: This study included 386 patients with hypertension. Mann-Whitney $U$ test and multivariate binary logistic regression analysis were used to investigate the relationship between NLR, CRP, BNP, and LVH in patients with hypertension, as well as compare the levels of NLR, CRP, and BNP in the four configurations. Receiver operator characteristic (ROC) curve was used to compare the diagnostic efficacy of NLR, CRP, and BNP on LVH. Results: The NLR and CRP and BNP levels of the LVH group were significantly higher than those of the non-LVH group. In the multivariate logistic regression analysis, NLR as well as age, BMI, and SBP were associated with LVH. In addition, in patients with eccentric and concentric hypertrophy, the NLR and CRP and BNP levels were higher than those of the normal left ventricular geometry and concentric remodeling groups. The cutoff values of NLR, CRP, and BNP obtained by ROC curve were 2.185, 2.205, and 283.45, respectively, for the prediction of LVH. Conclusions: NLR is independently associated with LVH in patients with hypertension, and this is consistent with the diagnostic efficacy of CRP and BNP, which may be a simple and convenient indicator for judging LVH.
\end{abstract}

Key words: CRP, BNP

(Int Heart J 2020; 61: 373-379)

$\mathrm{H}$ ypertension may cause severe organ damage and increase the risk of patient death and its incidence has been increasing year by year., ${ }^{1,2)}$ Left ventricular hypertrophy $(\mathrm{LVH})$ is a common pathological change in hypertension, which is a marker for and contributes to coronary events, heart failure, and cardiovascular mortality. ${ }^{3,4)}$ Inflammation, fibrosis, and oxidative stress as well as ischemia play significant roles and are the leading pathways in left ventricular remodeling. ${ }^{5)}$ It is well known that $\mathrm{C}$-reactive protein (CRP), brain natriuretic peptide (BNP), and some inflammatory factors play important roles in the progress of myocardial remodeling. Neutrophil-to-lymphocyte ratio (NLR) is a new inflammation marker in recent years, which is widely used in cardiovascular research. ${ }^{6-8)}$ Neutrophils, as phagocytic cells, are involved in the clearance of pathogens in infectious inflammation, and play an important role in tissue repair and immune regulation in aseptic inflammation. Lymphocytes participate in the long-term response of the immune system. NLR combines two different immune pathways and serves as a comprehensive indicator of inflammation, with a strong predictive ability for cardiovascular disease.
Studies have confirmed that elevated NLR is an independent risk factor of coronary atherosclerotic plaque progression, cardiac death after coronary stent implantation, and long-term mortality of acute coronary syndrome. ${ }^{9-14)}$ Although some studies have found that inflammatory cytokines (CRP, interleukin [IL]-6, IL-18, etc.) are associated with $\mathrm{LVH},{ }^{15,16)}$ no study clearly showed a relationship between NLR and LVH. We aimed to assess whether NLR is associated with LVH in patients with hypertension and to compare the diagnostic efficacy of NLR, CRP, and BNP for $\mathrm{LVH}$.

\section{Methods}

Study population: This study enrolled a total of 386 patients with hypertension admitted to the Department of Cardiology, Tianjin Medical University from July to September 2015. All enrolled patients signed informed consent forms. Hypertension was defined as systolic blood pressure $(\mathrm{SBP}) \geq 140 \mathrm{mmHg}$ and/or diastolic blood pressure (DBP) $\geq 90 \mathrm{mmHg}$ in the absence of antihypertensive drugs and when the blood pressure was measured

From the ${ }^{1}$ Department of Cardiology, Tianjin Medical University General Hospital, Tianjin, China.

*These authors contributed equally to this work.

This work was supported by Tianjin Health Planning Commission (No. 2015KZ117).

Address for correspondence: Yuemin Sun, MD, Department of Cardiology, Tianjin Medical University General Hospital, No. 154, AnShan Road, Tianjin, 300052, China. E-mail: zyysunyueminzr@126.com

Received for publication March 16, 2019. Revised and accepted August 12, 2019.

Released in advance online on J-STAGE March 14, 2020.

doi: 10.1536/ihj.19-138

All rights reserved by the International Heart Journal Association. 
three times a day. SBP $\geq 140 \mathrm{mmHg}$ and $\mathrm{DBP}<90$ $\mathrm{mmHg}$ was considered simple systolic hypertension. Patients with a history of hypertension and currently using antihypertensive drugs were also diagnosed as hypertensive even if the blood pressure was lower than 140/90 $\mathrm{mmHg}$. From these subjects, the patients who met the following conditions were excluded: (1) acute and chronic infection; (2) congenital heart disease; (3) severe heart failure; (4) myocardial infarction and cerebrovascular disease recently; (5) blood system diseases; (6) renal dysfunction and hepatic insufficiency; (7) rheumatic immune system diseases; (8) taking glucocorticoids, drugs that affect coagulation. In total, we enrolled 386 patients (aged $64.35 \pm 12.436$ years, including 201 males and 185 females).

Determination of laboratory indicators: The subjects were fasted for 10 hours and blood samples were drawn from the elbow vein. The blood samples were sent to the Laboratory Center of the General Hospital of Tianjin Medical University for the detection of blood routine item, BNP, creatine kinase, creatine kinase isoenzyme MB (CKMB), alanine aminotransferase, aspartate aminotransferase (AST), total cholesterol, triglycerides, high-density lipoprotein cholesterol, low-density lipoprotein cholesterol, creatinine, uric acid, fasting plasma glucose, CRP. All the tests were performed by professional laboratorians of the Tianjin Medical University General Hospital in strict accordance with instructions.

Echocardiography: Echocardiographic examination of the patients was performed by experienced cardiologist according to the guidelines of the American Society of Echocardiography. ${ }^{17)}$ The echocardiograph measurements included left ventricular internal dimension in end-diastole (LVIDd), interventricular septum thickness (IVST), posterior wall thickness (PWT). Left ventricular mass (LVM) $(\mathrm{g})=1.04 \times\left[(\text { LVIDd }+ \text { IVST }+ \text { PWT })^{3}-\right.$ LVDd $\left.^{3}\right]-13.6$ g. Left ventricular mass index (LVMI) $\left(\mathrm{g} / \mathrm{m}^{2}\right)=\mathrm{LVM}(\mathrm{g}) /$ BSA $\left(\mathrm{m}^{2}\right)$. Relative wall thickness $(\mathrm{RWT})=2 \times \mathrm{PWT} /$ LVIDd. LVH is defined as LVMI $\geq 125 \mathrm{~g} / \mathrm{m}^{2}$ in men and LVMI $\geq 120 \mathrm{~g} / \mathrm{m}^{2}$ in women. ${ }^{18)}$ A partition value of 0.45 was used for RWT. All subjects were divided into four configuration groups according to Ganau's classification as follows: normal left ventricular geometry (normal LVMI and RWT), concentric remodeling (normal LVMI and increased RWT), eccentric LVH (increased LVMI and normal RWT), and concentric LVH (increased LVMI and RWT).

Statistical analysis: Data analysis was performed with the SPSS 20.0 software. Continuous variables are expressed as mean $\pm \mathrm{SD}$ or quartile, and categorical variables are expressed as percentage. Comparisons among groups were made by chi-squared test for categorical variables. The $t$ test, Kruskal-Wallis test, and one-way ANOVA were used for continuous variables according to the number of groups and the distribution of the data. Multiple linear regression analysis was used to establish the relationship between NLR and LVH after the adjustment for gender, age, BMI, SBP, HR, CKMB, and AST. Receiver operator characteristic (ROC) curves were used to assess the diagnostic performance of NLR, CRP, and BNP. All $P$-values were the results of two-tailed tests. A value of $P<0.05$ indi- cated a statistically significant difference.

\section{Results}

Baseline characteristics, laboratory findings, and echocardiographic data in LVH and non-LVH groups: Patients were divided into two groups according to the 2010 Chinese guidelines for the management of hypertension, the LVH (190 patients) and non-LVH (196 subjects) groups. Table I shows the general characteristics between the two groups. Patients with LVH had higher CRP, AST, SBP, EF, BNP, CKMB, IVST, PWT, LVM, and LVMI compared with those of the non-LVH group $(P<0.05$ for all). The NLR level of the LVH group (2.635 [1.8983.590]) was significantly higher than that of the non-LVH group (2.190 [1.720-3.030]). The CRP level of the LVH group (2.555 [0.8-8.705]) was significantly higher than that of the non-LVH group (1.425 [0.6825-3.97]). The level of BNP of the LVH group (368 [88.325-1181]) was significantly higher than that of the non-LVH group (95.97 [52.91-271.775]) (Figure 1).

Multivariate logistic regression: In order to clarify the independent predictors of LVH, gender, age, BMI, SBP, $\mathrm{HR}, \mathrm{CKMB}$, and AST were included in the multivariate logistic regression. The result showed that NLR (odd ratio [OR]: $1.188 ; 95 \%$ confidence interval [CI]: 1.018-1.385; $P$ $=0.029)$, age (OR: $1.023 ; 95 \%$ CI: 1.004-1.041; $P=$ 0.016), BMI (OR: 1.075; 95\% CI: 1.009-1.144; $P=$ 0.024), and SBP (OR: 1.020; 95\% CI: 1.009-1.031; $P<$ 0.001) were independently associated with LVH (Table II).

NLR, CRP, and BNP levels were significantly increased in the eccentric $\mathrm{LVH}$ and concentric LVH groups compared with the normal left ventricular geometry and concentric remodeling groups: In order to clarify the relationship between NLR and LVH, we further divided the subjects into four groups according to their left ventricular configuration on the basis of LVMI and RWT as follows: a normal left ventricular geometry group, a concentric remodeling group, an eccentric LVH group and a concentric LVH group. Table III shows the general characteristics of the four groups. The results showed that NLR, and the levels of CRP and BNP were significantly increased in the eccentric LVH and concentric LVH groups compared with those of the normal left ventricular geometry and concentric remodeling groups (Figure 2).

The analysis of the ROC curve: The NLR cutoff value in the analysis of the ROC curve was 2.185 for predicting LVH (sensitivity: 68.9\%; specificity: $50 \%$ ), and the area under the ROC curve was $59.3 \%$ (95\% CI: $0.536-0.65 ; P$ $=0.002)$. The area under the ROC curve of CRP was 59.9\% (95\% CI: $0.542-0.655 ; P=0.001)$. The cutoff value was 2.205 , and the sensitivity and specificity were $55.3 \%$ and $61.7 \%$, respectively. The area under the ROC curve of BNP was $68.5 \%$ (95\% CI: 0.632-0.738; $P<$ $0.001)$. The cutoff value was 283.45 , and the sensitivity and specificity were $57.9 \%$ and $76 \%$, respectively (Figure 3 and Table IV). 
Table I. Clinical Characteristics of the Study Subjects in the Control and LVH Groups $(n=386)$

\begin{tabular}{|c|c|c|c|}
\hline & Control group & LVH group & $P$ \\
\hline$n$ & 196 & 190 & \\
\hline Males, $n(\%)$ & $101(51.53)$ & $100(52.63)$ & 0.829 \\
\hline Age, years & $64(58,71)$ & $66(58,75)$ & 0.057 \\
\hline Diabetes, $n(\%)$ & $59(30.1)$ & $48(25.26)$ & 0.288 \\
\hline Smoking, $n(\%)$ & $72(36.73)$ & $82(43.16)$ & 0.198 \\
\hline Atrial fibrillation, $n(\%)$ & $27(13.78)$ & $38(20)$ & 0.102 \\
\hline HR & $72(64,80.75)$ & $73.5(63,83)$ & 0.731 \\
\hline SBP & $140(121.25,150)$ & $142(130,157.25)$ & 0.003 \\
\hline DBP & $80(75,90)$ & $80(71.75,90)$ & 0.753 \\
\hline BMI, $\mathrm{kg} / \mathrm{m}^{2}$ & $25.7866 \pm 3.63106$ & $26.4108 \pm 3.58461$ & 0.09 \\
\hline CK & $72(51,112)$ & $84.5(52,165)$ & 0.073 \\
\hline CKMB & $10(7,16)$ & $12(8,18)$ & 0.021 \\
\hline ALT, U/L & $16(12,28)$ & $19(12,31)$ & 0.244 \\
\hline AST, U/L & $17(13,28)$ & $20.5(14,31)$ & 0.049 \\
\hline $\mathrm{TC}, \mathrm{mmol} / \mathrm{L}$ & $4.3923 \pm 0.9492$ & $4.4146 \pm 1.25981$ & 0.845 \\
\hline $\mathrm{TG}$, median, $\mathrm{mmol} / \mathrm{L}$ & $1.335(0.9625,1.87)$ & $1.31(0.99,1.8125)$ & 0.955 \\
\hline $\mathrm{HDL}, \mathrm{mmol} / \mathrm{L}$ & $1.015(0.85,1.2475)$ & $0.99(0.8275,1.2225)$ & 0.482 \\
\hline $\mathrm{LDL}, \mathrm{mmol} / \mathrm{L}$ & $2.6105 \pm 0.80069$ & $2.6958 \pm 1.03277$ & 0.366 \\
\hline $\mathrm{Scr}, \mathrm{mmol} / \mathrm{L}$ & $70(60,80)$ & $71(60.75,81.25)$ & 0.464 \\
\hline $\mathrm{UA}, \mu \mathrm{mol} / \mathrm{L}$ & $359.5 \pm 110.729$ & $366.66 \pm 107.395$ & 0.522 \\
\hline FPG, median, mmol/L & $5.25(4.7,6.0)$ & $5.25(4.8,6.125)$ & 0.506 \\
\hline CRP & $1.425(0.6825,3.97)$ & $2.555(0.8,8.705)$ & 0.001 \\
\hline BNP & $95.97(52.91,271.775)$ & $368(88.325,1181)$ & $<0.001$ \\
\hline NLR & $2.19(1.72,3.03)$ & $2.635(1.8975,3.59)$ & 0.002 \\
\hline $\mathrm{EF}$ & $63(60,64)$ & $60(51.75,64)$ & $<0.001$ \\
\hline IVST, mm & $10(9,11)$ & $11(10,12)$ & $<0.001$ \\
\hline PWT, mm & $10(9,10)$ & $11(10,12)$ & $<0.001$ \\
\hline RWT & $0.4167(0.3913,0.4634)$ & $0.4314(0.3846,0.4804)$ & 0.214 \\
\hline LVM, g & $187.425(157.8,216.19)$ & $253.02(220.52,290.72)$ & $<0.001$ \\
\hline LVMI, $\mathrm{g} / \mathrm{m}^{2}$ & $105.85(96.0875,114.4975)$ & $140.735(131.6225,157.4175)$ & $<0.001$ \\
\hline
\end{tabular}

Comparisons between groups were analyzed by chi-squared test for enumeration data (males, diabetes, smoking, and atrial fibrillation) and t-test for measurement data (remaining others). Data are mean \pm SD or percentage. LVH indicates left ventricular hypertrophy; HR, heart rate; SBP, systolic blood pressure; DBP, diastolic blood pressure; BMI, body mass index; Scr, serum creatinine; BNP, brain natriuretic peptide; CK, creatine kinase; CKMB, creatine kinase isoenzyme MB; ALT, alanine aminotransferase; AST, aspartate aminotransferase; TC, total cholesterol; TG, triglycerides; HDL-C, high-density lipoprotein cholesterol; LDL-C, low-density lipoprotein cholesterol; Scr, creatinine; UA, uric acid; FPG, fasting plasma glucose; CRP, C-reactive protein; EF, ejection fraction; LVIDd, left ventricular internal dimension in end-diastole; IVST, interventricular septum thickness; PWT, posterior wall thickness; RWT, relative wall thickness; LVM, left ventricular mass; and LVMI, left ventricular mass index.

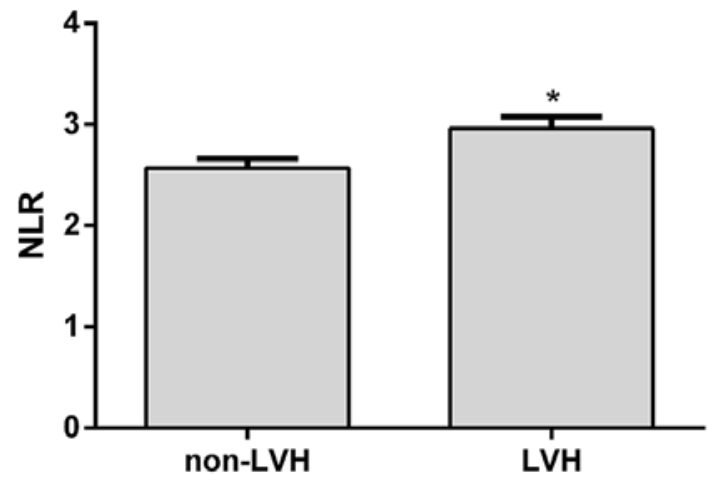

Figure 1. NLR levels between the non-LVH and LVH groups. $* P<$ 0.05 versus non-LVH group.
Table II. Multivariate Logistic Regression of Left Ventricular Hypertrophy in Hypertensive Patients

\begin{tabular}{lccccc}
\hline Variables & B & $P$ & OR & \multicolumn{2}{c}{ 95\% CI (lower-upper) } \\
\hline Gender (1) & -0.047 & 0.831 & 0.954 & 0.618 & 1.473 \\
Age & 0.022 & 0.016 & 1.023 & 1.004 & 1.041 \\
BMI & 0.072 & 0.024 & 1.075 & 1.009 & 1.144 \\
SBP & 0.020 & 0.000 & 1.020 & 1.009 & 1.031 \\
HR & 0.003 & 0.646 & 1.003 & 0.989 & 1.017 \\
CKMB & 0.01 & 0.055 & 1.010 & 1.000 & 1.019 \\
AST & 0.002 & 0.700 & 1.002 & 0.990 & 1.014 \\
NLR & 0.172 & 0.029 & 1.188 & 1.018 & 1.385 \\
\hline
\end{tabular}

OR indicates odds ratio; $\mathrm{CI}$, confidence interval; BMI, body mass index; SBP, systolic blood pressure; HR, heart rate; CKMB, creatine kinase isoenzyme MB; AST, aspartate aminotransferase; and NLR, neutrophil-to-lymphocyte ratio. 
Table III. Clinical Characteristics of the Study Subjects in Left Ventricular Geometry Pattern

\begin{tabular}{|c|c|c|c|c|c|}
\hline & Normal Lvgeometry & Concentric remodeling & Concentric LVH & Eccentric LVH & $P$ \\
\hline$n$ & 133 & 63 & 77 & 113 & \\
\hline Males, $n(\%)$ & $69(51.8)$ & $32(50.79)$ & $41(53.25)$ & $59(52.21)$ & 0.993 \\
\hline Age, years & $62.27 \pm 11.270$ & $64.17 \pm 14.995$ & $63.58 \pm 13.402$ & $66.83 \pm 11.140$ & 0.06 \\
\hline Diabetes, $n(\%)$ & $40(30.08)$ & $19(30.16)$ & $17(22.08)$ & $31(27.43)$ & 0.619 \\
\hline Smoking, $n(\%)$ & $52(39.10)$ & $20(31.75)$ & $31(40.26)$ & $51(45.13)$ & 0.38 \\
\hline Atrial fibrillation, $n(\%)$ & $16(12.03)$ & $11(17.46)$ & $14(18.18)$ & $24(21.24)$ & 0.748 \\
\hline HR & $72.73 \pm 14.72$ & $76.62 \pm 15.572$ & $73.44 \pm 11.904$ & $75.02 \pm 17.822$ & 0.35 \\
\hline SBP & $138.95 \pm 20.028$ & $137.02 \pm 17.214$ & $149.49 \pm 22.088$ & $140.96 \pm 18.455$ & $<0.001$ \\
\hline DBP & $80(71.5,90)$ & $80(79,90)$ & $80(74,95)$ & $80(71,90)$ & 0.393 \\
\hline BMI, $\mathrm{kg} / \mathrm{m}^{2}$ & $25.6725 \pm 3.6389$ & $26.0275 \pm 3.63158$ & $26.5073 \pm 3.33238$ & $26.3450 \pm 3.7651$ & 0.338 \\
\hline CK & $68(52.5,102)$ & $94(49,123)$ & $70(47.5,116)$ & $98(58.5,226)$ & 0.001 \\
\hline CKMB & $10(7,16)$ & $10(8,16)$ & $9(7,14)$ & $14(9,22)$ & $<0.001$ \\
\hline $\mathrm{ALT}, \mathrm{U} / \mathrm{L}$ & $17(12.5,29.5)$ & $15(11,24)$ & $16(12,25)$ & $19(13,35.5)$ & 0.125 \\
\hline AST, U/L & $17(13,29.5)$ & $18(13,22)$ & $17(13,25)$ & $23(15,45)$ & 0.003 \\
\hline $\mathrm{TC}, \mathrm{mmol} / \mathrm{L}$ & $4.3702 \pm 0.93080$ & $4.439 \pm 0.99292$ & $4.2071 \pm 0.94418$ & $4.556 \pm 1.42253$ & 0.194 \\
\hline $\mathrm{TG}$, median, $\mathrm{mmol} / \mathrm{L}$ & $1.33(0.97,1.89)$ & $1.34(0.94,1.87)$ & $1.42(0.96,1.91)$ & $1.29(1.01,1.78)$ & 0.89 \\
\hline $\mathrm{HDL}, \mathrm{mmol} / \mathrm{L}$ & $1.01(0.845,1.23)$ & $1.03(0.86,1.27)$ & $0.99(0.83,1.17)$ & $0.98(0.82,1.285)$ & 0.756 \\
\hline $\mathrm{LDL}, \mathrm{mmol} / \mathrm{L}$ & $2.5786 \pm 0.79121$ & $2.6778 \pm 0.82267$ & $2.5131 \pm 0.82003$ & $2.8203 \pm 1.14238$ & 0.093 \\
\hline $\mathrm{Scr}, \mathrm{mmol} / \mathrm{L}$ & $68(58,79.5)$ & $70(60,80)$ & $71(58,85.5)$ & $70(61,79)$ & 0.642 \\
\hline $\mathrm{UA}, \mu \mathrm{mol} / \mathrm{L}$ & $359.06 \pm 103.923$ & $360.44 \pm 124.796$ & $364.03 \pm 114.819$ & $368.49 \pm 102.423$ & 0.921 \\
\hline FPG, median, $\mathrm{mmol} / \mathrm{L}$ & $5.3(4.8,6.0)$ & $5.1(4.6,6.3)$ & $5.3(4.75,5.85)$ & $5.2(4.8,6.4)$ & 0.715 \\
\hline CRP & $1.53(0.705,4.705)$ & $1.21(0.60,3.5)$ & $2.1(0.67,4.955)$ & $3.08(1.125,10.455)$ & 0.001 \\
\hline BNP & $95.94(54.76,277.55)$ & $96(46.06,257.3)$ & $220.9(70.155,616.8)$ & $612.1(112.95,1945.5)$ & $<0.001$ \\
\hline NLR & $2.23(1.73,3.02)$ & $2.04(1.67,3.64)$ & $2.60(1.875,3.375)$ & $2.66(1.96,3.67)$ & 0.014 \\
\hline $\mathrm{EF}$ & $62(60,64)$ & $63(60,65)$ & $64(60.5,65)$ & $57(42,63)$ & $<0.001$ \\
\hline IVST, mm & $10(9,10)$ & $11(10,11)$ & $12(12,13)$ & $10(10,11)$ & $<0.001$ \\
\hline PWT, mm & $10(9,10)$ & $11(10,11)$ & $12(12,13)$ & $10(10,11)$ & $<0.001$ \\
\hline RWT & $0.4(0.3774,0.4176)$ & $0.4783(0.4651,0.5106)$ & $0.4898(0.4783,0.5217)$ & $0.4(0.3589,0.4211)$ & $<0.001$ \\
\hline LVM, g & $187.093 \pm 38.182$ & $189.624 \pm 38.684$ & $261.425 \pm 46.922$ & $264.118 \pm 60.748$ & $<0.001$ \\
\hline LVMI, $g / \mathrm{m}^{2}$ & $105.26(95.355,113.89)$ & $106.92(97.26,115.33)$ & $145.03(132.595,157.985)$ & $139.21(130.01,157.31)$ & $<0.001$ \\
\hline
\end{tabular}

Comparisons between groups were analyzed by ANOVA or Kruskal-Wallis test for measurement data and chi-squared test for enumeration data. Data are mean \pm SD or percentage. LV indicates left ventricle; LVH, left ventricular hypertrophy; HR, heart rate; SBP, systolic blood pressure; DBP, diastolic blood pressure; BMI, body mass index; Scr, serum creatinine; BNP, brain natriuretic peptide; CK, creatine kinase; CKMB, creatine kinase isoenzyme MB; ALT, alanine aminotransferase; AST, aspartate aminotransferase; TC, total cholesterol; TG, triglycerides; HDL-C, high-density lipoprotein cholesterol; LDL-C, low-density lipoprotein cholesterol; Scr, creatinine; UA, uric acid; FPG, fasting plasma glucose; CRP, C-reactive protein; EF, ejection fraction; LVIDd, left ventricular internal dimension in end-diastole; IVST, interventricular septum thickness; PWT, posterior wall thickness; RWT, relative wall thickness; BSA, body surface area; LVM, left ventricular mass; and LVMI, left ventricular mass index.

\section{Discussion}

The findings of the present study demonstrate that a simple ratio (NLR) obtained from blood routine examination provides relevant information regarding $\mathrm{LVH}$ in patients with hypertension. The level of NLR of the patients with LVH is higher than that of the normal configuration, and its diagnostic efficacy is similar to those of CRP and BNP; it can be used as a marker to judge the LVH.

In the past several years, it has become increasingly evident that hypertension is an inflammatory process that involves the transmigration and accumulation of both innate and adaptive immune cells into the interstitium of affected tissues, where they release cytokines and promote oxidative stress. ${ }^{19)}$ The immune system has two major components, the innate and the adaptive systems, which closely interact with each other. The innate immune response includes epithelial cells, which prevent the entrance of pathogens, professional phagocytes (neutrophils and macrophages), the complement system, and pattern recognition receptors. In contrast to the innate immune re- sponse, the adaptive immune response is designed to respond specifically to foreign antigens. In the case of T-cell activation, antigen-presenting cells degrade foreign proteins to small peptides that are presented by major histocompatibility complex proteins, produce cytokines, and alter the expression of surface receptors that lead to their transferring from secondary lymphoid organs to sites of peripheral inflammation. Studies have shown that angiotensin II (Ang II) and excessive catecholamines lead to the formation of effector-like $\mathrm{T}$ cells that infiltrate the perivascular regions of both large arteries and arterioles. There is also accumulation of monocyte/macrophages in these regions. Cytokines released from these cells promote vascular dysfunction and damage, leading to increased systemic vascular resistance. Reactive oxygen species are components of the innate immunity response, which activates proinflammatory transcription factors such as Nrf2 and NF- $\kappa B$. These, in turn, modulate the expression of genes encoding adhesion molecules and chemokines, and further promote inflammation. ${ }^{20-22)}$ Cardiac stress can induce morphological, structural, and functional changes of 

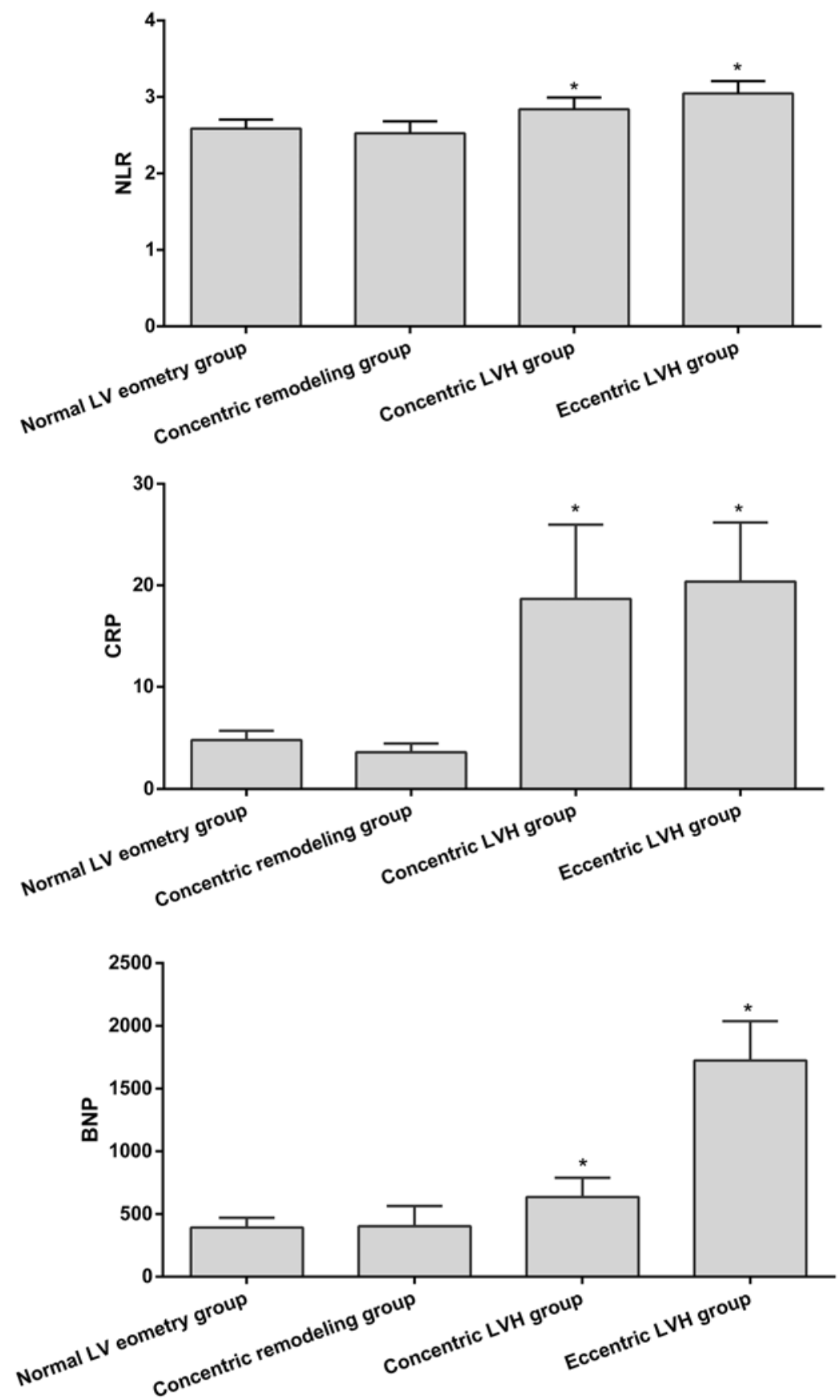

Figure 2. NLR and CRP and BNP levels in the four groups. $* P<0.05$.

the heart, which is referred to as cardiac remodeling. Sustained overload, as a result of pathological causes by hypertension, results in progressive heart remodeling and finally leads to heart failure. ${ }^{23)}$ Damage to the vascular endothelium occurs under long-time high impact of blood pressure, leading to the activation of the inflammatory response system, prompting the organizations and organs to produce a large amount of inflammatory cytokines. Inflammatory cells and cytokines participate in the pathological process of cardiovascular remodeling, and they can clear necrotic cells and foreign antigens as well as pro- mote angiogenesis and scar repair. Inflammatory factors are typically derived from white blood cells, particularly neutrophils. $^{24-26)}$

Recent studies have found that the reciprocal interaction between macrophages and $\mathrm{T}$ cells in the heart stimulates IFN-c expression, leading to increased MCP-1 expression in macrophages, which results in a forward-feed recruitment of macrophages, thus contributing to Ang IIinduced cardiac inflammation and fibrosis. ${ }^{27)} \mathrm{Wu}$, et al. reported that neutrophil-generated S100a8/S100a9 proteins are the key molecules that initiate Ang II-induced cardiac 


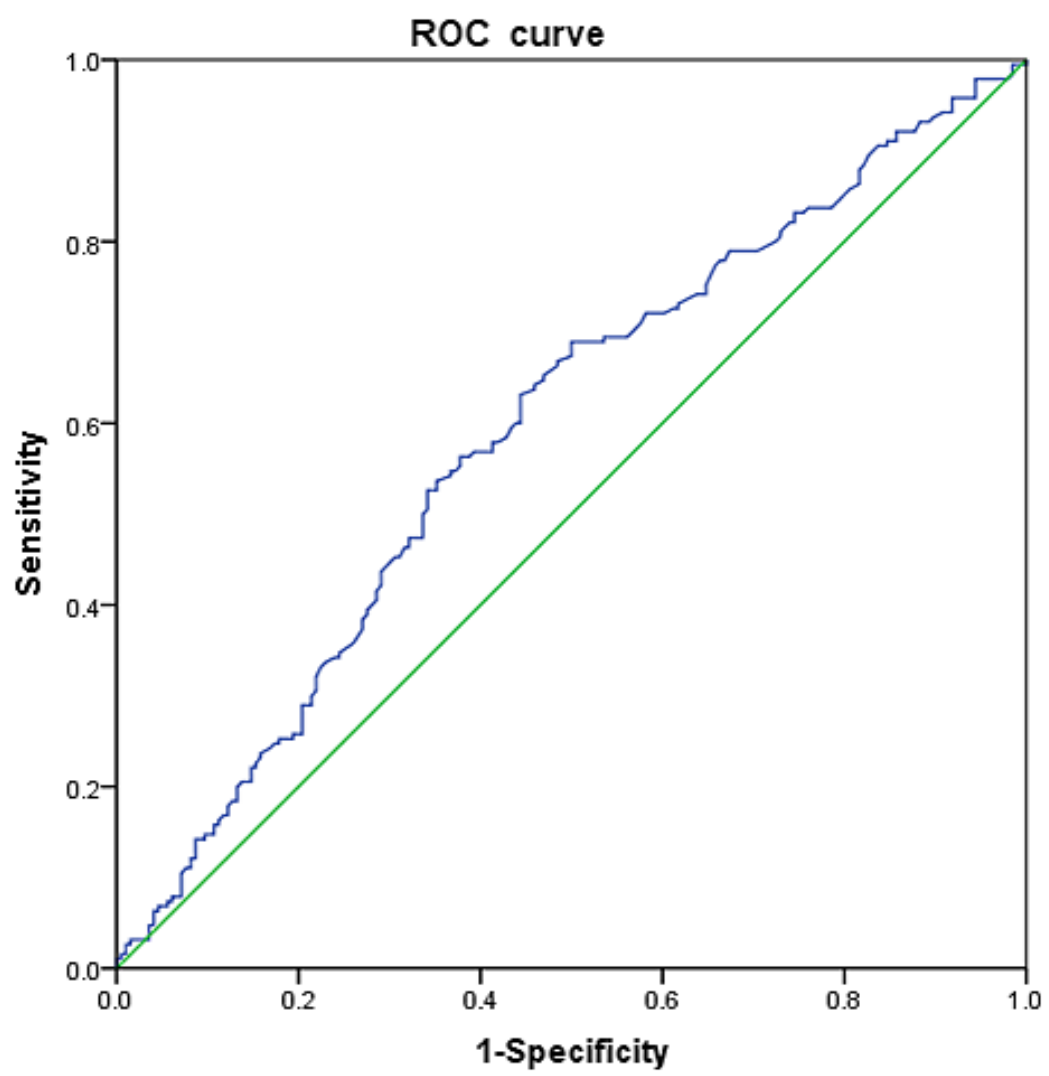

Figure 3. The receiver operator characteristic (ROC) curve analysis of NLR, CRP, and BNP for $\mathrm{LVH}$.

Table IV. ROC Curve Parameters of NLR, CRP, and BNP

\begin{tabular}{lccc}
\hline & NLR & CRP & BNP \\
\hline AUC & $59.30 \%$ & $59.90 \%$ & $68.50 \%$ \\
$95 \%$ CI & $0.536-0.65$ & $0.542-0.655$ & $0.632-0.738$ \\
$P$ & 0.002 & 0.001 & 0.001 \\
Cut-off & 2.185 & 2.205 & 283.45 \\
\hline
\end{tabular}

inflammation and fibrosis independently from the high blood pressure response. ${ }^{28)}$ Jiang, et al. reported that after infusion of Ang II to mice, elevated blood pressure, neutrophil accumulation, proinflammatory cytokine expression, reactive oxygen species production, and cardiac fibrosis occurred. ${ }^{29}$ Wei, et al. reported that the development of hypertension and LVH in patients with hypertension may be associated with the apoptosis in lymphocytes. The levels of apoptosis of lymphocytes in the hypertensive group $(65 \%)$ was significantly higher than that of the control group (12.5\%). The lymphocyte apoptosis rate of the LVH group $(33.1 \%)$ was significantly higher than that of the non-LVH group $(12.43 \% ; P<0.05){ }^{30)}$ ÖZzbïçer, et al. reported that the level of LVMI could be predicted by IL-18 level independently in both the general population and the newly diagnosed patients with hypertension. ${ }^{15)}$ Salles, et al. found that LVH was associated with microalbuminuria and CRP levels. ${ }^{31}$

$\mathrm{BNP}$, a cardiac neurohormone, is released in response to increased left ventricular wall stretch. ${ }^{14)} \mathrm{BNP}$ is associated with cardiac remodeling in patients with hypertension. ${ }^{32)} \mathrm{CRP}$, the most investigated cytokine in hypertension, has been repeatedly implicated in both the initiation and progression of the disorder. ${ }^{33)}$ NLR, an indicator of systemic inflammation, has been proposed as a useful biomarker to predict cardiovascular risk and events. ${ }^{6}$ Karagöz, et al. reported that NLR was a predictor of diastolic dysfunction in patients with hypertension. ${ }^{34)}$ Shi, et al. reported that white blood cell count may be associated with LVH in patients with hypertension currently taking antihypertensive drugs. NLR may be useful for predicting left VR in patients with ST elevation myocardial infarction after primary percutaneous coronary intervention. ${ }^{14)}$ Suliman, et al. reported that NLR is clearly an independent predictor of all-cause mortality in patients with ACS. ${ }^{35}$

In conclusion, NLRs were significantly increased in the LVH patients. NLR, as a simple, relatively inexpensive, and universally available test may become the indicator of LVH, which can facilitate the monitoring of cardiac remodeling and taking early treatment measures to reduce the incidence of heart failure

There were some limitations to our study. The number of samples was relatively small and the conclusions drawn from our study should be tested with a larger sample size. Furthermore, our study is a cross-sectional study that would be more valuable for assessing the relationship between NLR and hypertension if we could have 
followed-up the prognosis of these patients. Since previous reports already suggested the importance of inflammatory cytokines, we will make efforts to measure cytokines in the future study. We should also select healthy subjects without hypertension in the future for control.

\section{Disclosure}

Conflicts of interest: The authors declare that they have no competing interests.

\section{References}

1. Westerlund E, Brandt L, Hovatta O, Wallén H, Ekbom A, Henriksson $\mathrm{P}$. Incidence of hypertension, stroke, coronary heart disease, and diabetes in women who have delivered afterinvitro fertilization: a population-based cohort study from Sweden. Fertil Steril 2014; 102: 1096-102

2. Veloso HH. Incidence of sudden cardiac death in congestive heart failure: Chagas disease versus systemic arterial hypertension. Int J Cardiol 2014; 175: 175-6.

3. Desai CS, Ning H, Lloyd-Jones DM. Competing cardiovascular outcomes associated with electrocardiographic left ventricular hypertrophy: the Atherosclerosis Risk in Communities Study. Heart 2012; 98: 330-4.

4. Messerli FH, Aepfelbacher FC. Hypertension and left-ventricular hypertrophy. Cardiol Clin 1995; 13: 549-57.

5. Tin LL, Beevers DG, Lip GY. Hypertension, left ventricular hypertrophy, and sudden death. Curr Cardiol Rep 2002; 4: 449-57.

6. Horne BD, Anderson JL, John JM, et al. Which white blood cell subtypes predict increased cardiovascular risk? J Am Coll Cardiol 2005; 45: 1638-43.

7. Papa A, Emdin M, Passino C, Michelassi C, Battaglia D, Cocci F. Predictive value of elevated neutrophil-lymphocyte ratio on cardiac mortality in patients with stable coronary artery disease. Clin Chim Acta 2008; 395: 27-31.

8. Bhat T, Teli S, Rijal J, et al. Neutrophil to lymphocyte ratio and cardiovascular diseases: a review. Expert Rev Cardiovasc Ther 2013; 11: 55-9.

9. Kalay N, Dogdu O, Koc F, et al. Hematologic parameters and angiographic progression of coronary atherosclerosis. Angiology 2012; 63: 213-7.

10. Turak O, Ozcan F, Isleyen A, et al. Usefulness of the neutrophil-to-lymphocyte ratio to predict bare-metal stent restenosis. Am J Cardiol 2012; 110: 1405-10.

11. Muhmmed Suliman MA, Bahnacy Juma AA, Ali Almadhani AA, Pathare AV, Alkindi SS, Uwe Werner F. Predictive value of neutrophil to lymphocyte ratio in outcomes of patients with acute coronary syndrome. Arch Med Res 2010; 41: 618-22.

12. Shyam P, Erdal C, Waqas K, Marmur JD. Neutrophil to lymphocyte ratio as a predictor of long-term mortality in African Americans undergoing percutaneous coronary intervention. Clin Cardiol 2010; 32: E6-10.

13. Gibson PH, Croal BL, Cuthbertson BH, et al. Preoperative neutrophil-lymphocyte ratio and outcome from coronary artery bypass grafting. Am Heart J 2007; 154: 995-1002.

14. Börekçi A, Gür M, Türkoğlu C, et al. Neutrophil to lymphocyte ratio predicts left ventricular remodeling in patients with ST Elevation Myocardial infarction after primary percutaneous coronary intervention. Korean Circ J 2016; 46: 15-22.

15. ÖZzbïçer S, Uluçam ZM. Association between interleukin-18 level and left ventricular mass index in hypertensive patients. Korean Circ J 2017; 47: 238-44.

16. Masiha S, Sundström J, Lind L. Inflammatory markers are associated with left ventricular hypertrophy and diastolic dysfunc- tion in a population-based sample of elderly men and women. J Hum Hypertens 2013; 27: 13-7.

17. Gerhard-Herman M, Gardin JM, Jaff M, et al. Guidelines for noninvasive vascular laboratory testing: a report from the American Society of Echocardiography and the Society for Vascular Medicine and Biology. J Am Soc Echocardiogr 2006; 19: 955-72.

18. Slyter H. Guidelines for the management of patients with acute ischemic stroke. Stroke 1995; 26: 137-8.

19. Harrison DG, Guzik TJ, Lob HE, et al. Inflammation, immunity, and hypertension. Hypertension 2011; 57: 132-40.

20. Rodríguez-Iturbe B, Pons H, Quiroz Y, Johnson RJ. The immunological basis of hypertension. Am J Hypertens 2014; 27 : 1327-37.

21. De Miguel C, Rudemiller NP, Abais JM, Mattson DL. Inflammation and hypertension: new understandings and potential therapeutic targets. Curr Hypertens Rep 2015; 17: 507.

22. Mcmaster WG, Kirabo A, Madhur MS, Harrison DG. Inflammation, immunity, and hypertensive end-organ damage. Circ Res 2015; 116: 1022-33.

23. Piek A, de Boer RAD, Silljé HHW. The fibrosis-cell death axis in heart failure. Heart Fail Rev 2016; 21: 199-211.

24. Fanning NF, Kell MR, Shorten GD, et al. Circulating granulocyte macrophage colony-stimulating factor in plasma of patients with the systemic inflammatory response syndrome delays neutrophil apoptosis through inhibition of spontaneous reactive oxygen species generation. Shock 1999; 11: 167-74.

25. Vanderslice P, Munsch CL, Rachal E, et al. Angiogenesis induced by tumor necrosis factor-agr; is mediated by $\alpha 4$ integrins. Angiogenesis 1998; 2: 265-75.

26. Wright CR, Brown EL, Della Gatta PA, et al. Regulation of granulocyte colony-stimulating factor and its receptor in skeletal muscle is dependent upon the type of inflammatory stimulus. J Interferon Cytokine Res 2015; 35: 710-9.

27. Han YL, Li YL, Jia LX, et al. Reciprocal interaction between macrophages and $\mathrm{T}$ cells stimulates IFN- $\gamma$ and MCP- 1 production in Ang II-induced cardiac inflammation and fibrosis. PLOS One 2012; 7: e35506.

28. Wu Y, Li Y, Zhang C, et al. S100a8/a9 released by CD11b+Gr1 + neutrophils activates cardiac fibroblasts to initiate angiotensin II-Induced cardiac inflammation and injury. Hypertension 2014; 63: $1241-50$

29. Jiang HM, Wang HX, Yang H, et al. Role for granulocyte colony stimulating factor in angiotensin II-induced neutrophil recruitment and cardiac fibrosis in mice. Am J Hypertens 2013; 26: 1224-33.

30. Wei L, Yang L, Feng QI. Apoptosis of lymphocyte in hypertensive patients. Chin J Hypertens 2002; 2002: 204-6.

31. Salles GF, Fiszman R, Cardoso CR, Muxfeldt ES. Relation of left ventricular hypertrophy with systemic inflammation and endothelial damage in resistant hypertension. Hypertension 2007; 50: 723-8.

32. Phelan D, Watson C, Martos R, et al. Modest elevation in BNP in asymptomatic hypertensive patients reflects sub-clinical cardiac remodeling, inflammation and extracellular matrix changes. PLOS ONE 2012; 7: e49259.

33. Montecucco F, Pende A, Quercioli A, Mach F. Inflammation in the pathophysiology of essential hypertension. J Nephrol 2011; 24: 23-34.

34. Karag ZA, Vural A, Günayd NZY, et al. The role of neutrophil to lymphocyte ratio as a predictor of diastolic dysfunction in hypertensive patients. Eur Rev Med Pharmacol Sci 2015; 115: S10-1.

35. Muhmmed Suliman MA, Bahnacy Juma AA, Ali Almadhani AA, Pathare AV, Alkindi SS, Uwe Werner F. Predictive value of neutrophil to lymphocyte ratio in outcomes of patients with acute coronary syndrome. Arch Med Res 2010; 41: 618-22. 